\title{
Identification of a Novel c-Myc Inhibitor
} 7594-0037 by Structure-Based Virtual Screening and Investigation of Its Anti-Cancer Effect on Multiple Myeloma

This article was published in the following Dove Press journal:

Drug Design, Development and Therapy

\author{
Ruosi Yao ${ }^{1,2}$ \\ Yu Xie' \\ Xiaoyang Sun' \\ Menghui Zhang' \\ Jian Zhou' \\ Linlin $\mathrm{Liu}^{3}$ \\ Jian $\mathrm{Gao}^{4}$ \\ Kailin Xu (iD) ${ }^{1,2}$ \\ 'Blood Diseases Institute, Xuzhou \\ Medical University, Xuzhou, Jiangsu, \\ People's Republic of China; ${ }^{2}$ Department \\ of Hematology, The Affiliated Hospital of \\ Xuzhou Medical University, Xuzhou, \\ Jiangsu, People's Republic of China; \\ ${ }^{3}$ College of Medical Imaging, Xuzhou \\ Medical University, Xuzhou, Jiangsu, \\ People's Republic of China; ${ }^{4}$ Jiangsu Key \\ Laboratory of New Drug Research and \\ Clinical Pharmacy, Xuzhou Medical \\ University, Xuzhou, Jiangsu, People's \\ Republic of China
}

Introduction: Multiple myeloma (MM) is an extremely malignant and incurable hematological cancer. Increased expression of the c-Myc oncoprotein is closely associated with shorter overall survival of MM patients, implying that c-Myc is a potential therapeutic target. Main Methods: We identified a potential c-Myc inhibitor 7594-0037 by structure-based virtual screening from the ChemDiv database. CCK8 assay and flow cytometry were used to detect MM cell viability, cell cycle and apoptosis. Q-PCR and Western blot were used to measure corresponding mRNA and protein expression levels. Protein stability assay measured the stability of c-Myc.

Results: Compound 7594-0037 exhibited stronger anti-proliferative activity against MM cells, and induced MM cell cycle G2 phase arrest and apoptosis. More importantly, compound 7594-0037 overcame myeloma resistance to bortezomib and exhibited a synergistic effect with bortezomib, resulting in increased MM cell death. The mechanism consists of compound 7594-0037 facilitating c-Myc protein degradation via decreasing the c-Myc S62 phosphorylation levels mediated by PIM1 kinase. Molecular dynamics simulation with the c-Myc/7594-0037 complex showed that compound 7594-0037 bound tightly to the $\mathrm{N}$-terminus of c-Myc, and blocked the binding interaction of the two termini of c-Myc, which resulted in c-Myc entering into an unstable state.

Conclusion: Overall, our study provides preliminary data for compound 7594-0037, which can be used as a novel c-Myc inhibitor and is a potential candidate therapeutic drug for multiple myeloma.

Keywords: multiple myeloma, c-Myc inhibitor, virtual screening, drug resistance, apoptosis

\section{Introduction}

The c-Myc protein is an important basic-helix-loop-helix leucine zipper (bHLH-LZ) transcriptional factor that plays a key role in proliferation, differentiation, metabolism, and angiogenesis. ${ }^{1-3}$ Although c-Myc is crucial for normal cell function, its abnormal expression is also closely associated with various tumors, including breast, prostate, and colon cancers, and lymphoma and leukemia. ${ }^{4-7}$ Recently, gene expression analysis from 101 multiple myeloma (MM) patients confirmed that $c-M y c$ was activated in $67 \%$ of myeloma cases. ${ }^{8}$ Additionally, a retrospective study of $117 \mathrm{MM}$ patients indicated that overexpression of $c-M y c$ was found in $40 \%$ of MM samples, and ectopic expression of $c-M y c$ was also correlated with shorter overall survival of MM patients. ${ }^{9}$
Correspondence: Kailin Xu University, Xuzhou, Jiangsu, People's

Republic of China

Email lihmd@I63.com

Jian Gao

Jiangsu Key Laboratory of New Drug

Research and Clinical Pharmacy, Xuzhou

Medical University, Xuzhou, Jiangsu,

People's Republic of China

Email 18626042662@I63.com 
Thus, the c-Myc oncoprotein could be a potential therapeutic target for multiple myeloma.

Depletion of $c-M y c$ could lead to cancer cell cycle arrest, cell apoptosis, and tumor suppression. It has been reported that many small molecular c-Myc inhibitors impede c-Myc protein function, such as 10058-F4 and 10074-G5, which exhibited low micromolar IC50 values in vitro by targeting the interaction between c-Myc and MAX. ${ }^{10} \mathrm{JQ1}$, which is another c-Myc inhibitor, also decreased the amount of c-Myc protein by inhibiting BRD4, which is one of its coactivators. ${ }^{11}$ Thus, the existing c-Myc inhibitors exhibit a certain amount of effectiveness, but they do not directly disturb the c-Myc protein. Therefore, the pursuit of novel direct targeting of c-Myc with small molecular inhibitors is urgently required.

c-Myc is a highly unstable nuclear oncoprotein that can be regulated through multiple signaling pathways via transcriptional regulation and post-translational modification. ${ }^{7,12,13}$ PIM1, as a member of the PIM serine/threonine kinase family, phosphorylates c-Myc at serine 62 (S62), which increases c-Myc stability, thereby enhancing its transcriptional activity. ${ }^{14}$ However, c-Myc is also phosphorylated at threonine 58 (T58) by GSK3 $\beta$, which decreases c-Myc protein stability. ${ }^{15}$ Additionally, the ubiquitin-proteasome pathway is one of the most prominent mechanisms for c-Myc degradation. ${ }^{16}$ Hence, c-Myc can be easily and rapidly degraded once it is dephosphorylated at S62.

In this study, we carried out a structure-based virtual screening approach to develop potential c-Myc inhibitor from the ChemDiv database. Compound 7594-0037 directly disturbs the stability of the c-Myc protein by decreasing the c-Myc S62 phosphorylation level. We present evidence that compound 7594-0037 decreases MM cell viabilities in a dose- and time-dependent manner. More importantly, further enhancement of the killing effect on MM cells was achieved when compound 7594-0037 was combined with bortezomib. Herein, we characterized compound 7594-0037 to examine whether it indeed affected c-Myc protein stability and tested the idea that this novel c-Myc inhibitor can be used against $\mathrm{MM}$ and other hematological malignant cells.

\section{Materials and Methods}

\section{Structure-Based Virtual Screening}

To identify more potent c-Myc inhibitors, molecular docking based virtual screening was performed on the targeted protein c-Myc via the Surflex docking module in Sybyl-
X2.1 (Tripos Associates, St. Louis, MO, USA). The crystal structure of c-Myc-Max recognizing DNA (Protein Data Bank (PDB) ID: $1 \mathrm{NKP}^{17}$ ) for virtual screening was obtained from the RCSB PDB and the ChemDiv database from TopScience Co., Ltd. (Shanghai, China) was set as the ligand source. In light of only 2D-structural information was available, all compounds in the ChemDiv database were preprocessed by using the $\mathrm{db}$ translate module in Sybyl-X2.1. During the preparation of the receptor, the region Arg363-Ile381 was set as the active site and all water molecules were removed. To accelerate the virtual screening, a high-speed screening was firstly carried out by decreasing the maximum quantity of conformations and rotatable bonds from 20 to 10 , and from 100 to 50, respectively. Then, the molecules with a docking score within the top $1 \%$ were screened once more using the default docking parameters. Finally, several commercially available compounds were selected by ranking the docking scores and clustering analysis and purchased for the following in vitro biological activity assay.

\section{Cell Culture}

Human multiple myeloma cell lines Roswell Park Memorial Institute (RPMI)-8226 and U266 were purchased from the American Type Culture Collection (Manassas, VA, USA) and were cultured in RPMI-1640 medium supplemented with $10 \%$ fetal bovine serum (FBS). The RPMI-8226/BTZ100 cell line was kindly provided by Dr. Jacqueline Cloos (VU University Medical Center, The Netherlands). ${ }^{18}$

\section{Cell Viability Assay}

The indicated MM cells were seeded at 10,000/well in 100 $\mu \mathrm{L}$ RPMI-1640 medium in a 96-well plate. Then, cells were treated with different doses of compound 7594-0037 for different times at a given concentration of the c-Myc inhibitor. Eventually, cell survival rates were measured using a Cell Counting Kit (CCK)-8 kit according to the manufacturer's protocol. ${ }^{19,20}$

\section{Flow Cytometry}

The experiment was performed basically as previously described. ${ }^{21}$ Briefly, the indicated MM cells were treated with different doses of c-Myc inhibitor 7594-0037 for 48 $\mathrm{h}$ and harvested. For the cell cycle assay, the cells were washed with phosphate-buffered saline (PBS) and fixed overnight in $70 \%$ cold ethanol. The fixed cells were resuspended and incubated with RNase A, and then stained 
in PBS containing propidium iodide (PI) at room temperature. Finally, the cellular DNA was examined using a FACScan cell sorter and FACSCalibur flow cytometry (BD Biosciences). Early apoptotic cell death was examined with the Annexin V- fluorescein isothiocyanate (FITC)/PI Apoptosis Detection Kit following protocols from previous reports. ${ }^{22-24}$

\section{Western Blot}

Proteins were separated by sodium dodecyl sulfatepolyacrylamide gel electrophoresis (SDS-PAGE) and transferred to polyvinylidene difluoride (PVDF) membranes, which were blocked with $5 \%$ nonfat milk and incubated with anti- $\beta$-actin, anti-PARP1, anti-c-Myc, anti-pS62 -c-Myc, anti-pT58-c-Myc, and anti-PIM1 (Proteintech); and anti-caspase-3 and anti-caspase-9 (Cell Signaling Technology). Finally, the membranes were visualized with an enhanced chemiluminescence (ECL) system.

\section{Q-PCR Assay}

Total RNA was extracted with TRIzol reagent, and $1.5 \mu \mathrm{g}$ RNA was reverse transcribed into cDNA as previously described. ${ }^{25}$ Real-time quantitative PCR was performed using SYBR Green mix, and the results were normalized to the amplification of $\beta$-actin and to determination of the fold change based on $2-\Delta \Delta \mathrm{Ct}$.

\section{Protein Stability Assay}

The indicated MM cells were treated with c-Myc inhibitor 7594-0037 for different times ( $12 \mathrm{~h}, 24 \mathrm{~h}$, and $48 \mathrm{~h}$ ). The protein synthesis inhibitor cycloheximide (CHX) and proteasome inhibitor MG132 were used for analyzing protein stability as previously described. ${ }^{26}$

\section{Molecular Dynamics (MD) Simulation with c-Myc Inhibitor 7594-0037}

To study the molecular mechanism of compound 7594-0037 disrupting c-Myc protein stability, a 20-ns MD simulation was performed on c-Myc complexed with 7594-0037 using the Amber 12 molecular simulation package. $^{27}$ The initial structure of the complex was obtained from the molecular docking based virtual screening. A single c-Myc protein was also studied as a control. The standard amber force field (ff03) and general amber force field (GAFF) were used as the parameters for protein and ligand, respectively. Compound 7594-0037 was firstly minimized using the $\mathrm{HF} / 6-31^{*}$ optimization in the
Gaussian 09 program, ${ }^{28}$ and then the partial atomic charge was generated by fitting the electrostatic potential derived by the restrained electrostatic potential (RESP) fitting technique in Amber 12. Finally, the partial charge and GAFF force field parameter for compound 7594-0037 were obtained using the antechamber module in Amber 12.

Each system was placed into a cubic TIP3P water box, which extended $12 \AA$ from any solute atoms. Appropriate amounts of $\mathrm{Na}+$ were added to neutralize the systems. Energy minimizations of the systems were conducted by the Sander program in Amber 12 via three steps as described in our previous studies. ${ }^{29-31}$ Afterwards, each system was gradually warmed from 0 to $310 \mathrm{~K}$ over $60 \mathrm{ps}$ in the NVT ensemble. Under a constant temperature of 310 $\mathrm{K}$, a 20-ns MD simulation with a time step of 2 fs was carried out. In the process of sampling, the coordinates were saved every 1 ps.

\section{Statistical Analysis}

The data from all the above experiments are presented as the mean \pm SD from three independent experiments. Differences between different groups were analyzed using Student's $t$-test. $\mathrm{P}<0.05$ was considered statistically significant. The statistical analyses were performed using GraphPad Prism 5 (GraphPad Software, La Jolla, CA, USA).

\section{Results and Discussion}

\section{Structure-Based Virtual Screening and Binding Mode of Compound 7594-0037}

10 commercially available compounds (Figure S1) were selected from the two rounds of high-throughput virtual screenings by ranking the docking scores and clustering analysis. In light of the fact that potent c-Myc inhibitors should possess appropriate ant-proliferative activity in cancer cells, the hits obtained from the virtual screening were firstly evaluated in vitro cancer cell growth inhibition. Only compound 7594-0037 (Figure 1A) exhibited potent cytotoxic effect on MM cells (described as follows).

To study the binding mode of compound 7594-0037, the docked conformation of the c-Myc/7594-0037 complex is shown in Figure 1B. Compound 7594-0037 formed three hydrogen bonds with the residues Arg911, Arg914, and Asn915, and at the same time, was well accommodated in a hydrophobic cleft composed of residues Leu917, Lys918, Phe921, and Phe922. It is noteworthy that the indole moiety 
A

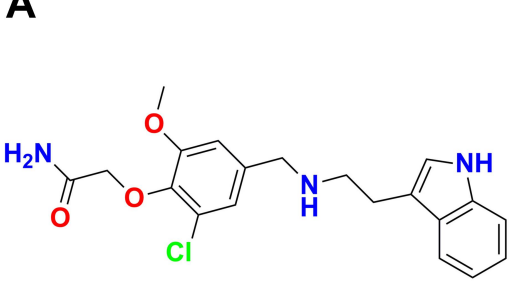

7594_0037
B

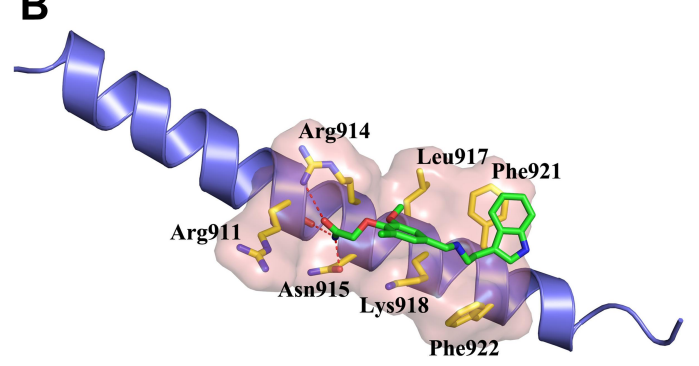

Figure I New c-Myc inhibitor 7594-0037. (A) Structure of compound 7594-0037. (B) The predicted the binding mode of compound 7594-0037 to its receptor c-Myc. The key residues of c-Myc that are used by compound 7594-0037 for binding are shown in stick model and colored in yellow.

of compound 7594-0037 had strong $\pi-\pi$ interaction with Phe921. Thus, the binding of compound 7594-0037 to c-Myc protein was dominated by both hydrogen bond interactions and hydrophobic interactions.

\section{Compound 7594-0037 Inhibited the Proliferation of MM Cells}

To determine the effect of the hits obtained from virtual screening on MM cell proliferation, RPMI-8226 and U266 cells were treated with different concentrations of compounds. The results showed that only compound 7594-0037 decreased MM cell viabilities in a dosedependent manner (Figure 2A and 2B). The IC50 value of compound 7594-0037 was determined in the $10-40 \mu \mathrm{M}$ range and calculated as $17.8 \mu \mathrm{M}$ for RPMI8226 and $27.9 \mu \mathrm{M}$ for U266 cells. It was reported that the small molecular inhibitor 10058-F4 specifically inhibits c-Myc-Max heterodimerization to further prevent transactivation of c-Myc target genes. $^{32,33}$ Nevertheless, 10085-F4 was insensitive to several myeloma cell lines, which had IC50 $>50 \mu \mathrm{M}$ and $<100$ $\mu \mathrm{M}^{34}$ Our c-Myc inhibitor compound 7594-0037 showed stronger antiproliferative activity than 10058F4, implying that compound 7594-0037 could be an excellent addition to the treatment options for MM patients. Additionally, we further observed that compound 7594-0037 time-dependently decreased MM cell viabilities (Figure 2C and D). Subsequently, we detected the effect of compound 7594-0037 on cell subpopulation distribution by flow cytometry. The results showed that compound 7594-0037 induced G2 phase arrest of MM cells in a dose-dependent manner (Figure 2D and E), suggesting that cell cycle arrest induced by compound 7594-0037 might at least cause partial decreased MM cell survival rates.

\section{Compound 7594-0037 Triggered MM Cell Death}

Next, we examined the effect of compound 7594-0037 on cell apoptosis of RPMI-8226 cells. By using flow cytometry based on Annexin V/PI staining, we found that c-Myc inhibitor 7594-0037 dose-dependently increased the ratio of apoptotic RPMI-8226 cells from $2.5 \%$ to $34.6 \%$, as compared with the DMSO control (Figure 3A and B). Similarly, we found that compound 7594-0037 also triggered U266 cell death in a concentration-dependent manner (Figure 3C). To further investigate the c-Myc inhibitorinduced MM cell apoptosis, we determined the activation of caspase family proteins using Western blot assay. As shown in Figure 3D, we found that compound 7594-0037 obviously promoted the cleavage of PARP1, caspase-9, and caspase- 8 pro-apoptotic associated proteins in RPMI-8226 and U266 cells. Owing to better antiproliferative activity than commercial c-Myc inhibitor 10058-F4, implying that compound 7594-0037 could be an excellent addition to the treatment options for MM patients. Subsequently, we further analyze the anti-tumor activity of compound 7594-0037 using xenograft model of myeloma. Unfortunately, our results demonstrated that compound 7594-0037 did not decrease primary MM tumor growth evidently (data not shown). Taken together, our data indicate that novel c-Myc inhibitor compound 7594-0037 triggers caspase-mediated myeloma cell apoptosis.

\section{Compound 7594-0037 Exhibited a Synergistic Effect in Combination with Bortezomib}

The introduction of bortezomib (BTZ) was an important breakthrough in the treatment of MM. ${ }^{35}$ However, BTZ resistance decreases the clinical effectiveness of MM treatments, and thus, it is critical to search for novel effective 
A

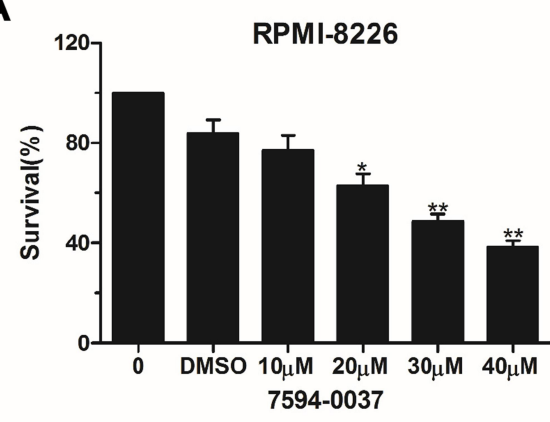

C

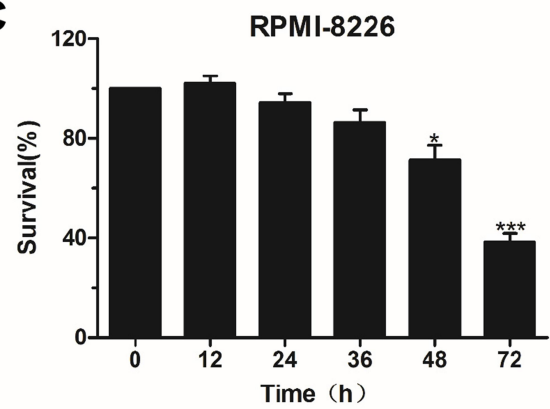

E

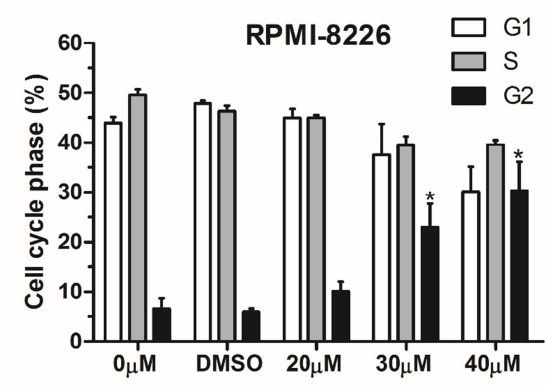

B

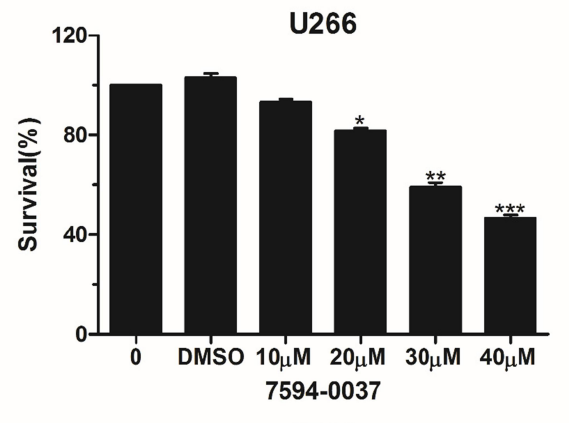

D $\quad 120 \quad$ U266

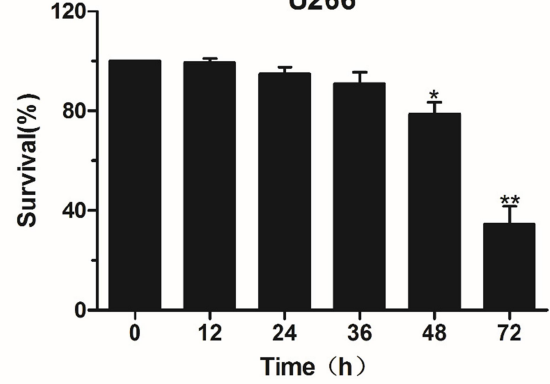

$F$

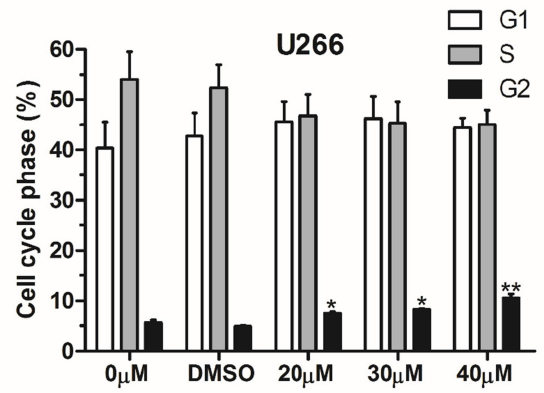

Figure 2 The effect of compound 7594-0037 on cell viability and cell cycle. (A-B) Cell viability analysis of RPMI-8226 and U266 cells treated with different concentrations of compound 7594-0037. (C-D) RPMI-8226 and U266 cells were treated with compound 7594-0037 at different time intervals, and the CCK-8 assay was performed to determine the cell survival rate. (E-F) The distribution of RPMI-8226 and U266 cell subpopulations treated with compound $7594-0037$ was analyzed by flow cytometry. The experiments were conducted at least three times. Error bars: mean $\pm \mathrm{SD} ;{ }^{* \mathrm{P}}<0.05 ; * * \mathrm{P}<0.0 \mathrm{I} ; * * * \mathrm{P}<0.00 \mathrm{I}$.

regimens to overcome BTZ resistance. In this study, the cytotoxic impact of compound 7594-0037 was examined using RPMI-8226 cells resistant to bortezomib. We conducted a cell viability and apoptosis assay using $100 \mathrm{nM}$ bortezomib-resistant MM cells (RPMI-8226/BTZ100) that have previously been described. ${ }^{18}$ Besides, we also established another $100 \mathrm{nM}$ bortezomib-resistant cell based on U266 MM cell line. Compound 7594-0037 decreased the RPMI-8226/BTZ100 and U266/BTZ100 cell viabilities in a concentration-dependent manner (Figure 4A). However, we found that the RPMI-8226/BTZ100 and U266/BTZ100 cells were inhibited in a time-independent manner (Figure 4B). We then wondered whether compound 7594-0037 had an effect on BTZ-resistant cell apoptosis. To confirm this, RPMI-8226/BTZ100 cells treated with compound 7594-0037 were subjected to apoptotic analysis by flow cytometry after staining with Annexin V/PI. As shown in Figure 4C and D, c-Myc inhibitor 7594-0037 increased the Annexin V+ cell fraction in RPMI-8226/ BTZ100 cells. Further flow cytometry assays showed that compound 7594-0037 exhibited a synergistic effect in combination with bortezomib in RPMI-8226 cells (Figure 4E), implying that novel c-Myc inhibitor compound 7594-0037 could overcome bortezomib myeloma resistance.

\section{Compound 7594-0037 Disturbed c-Myc Protein Stability}

We used structure based virtual screening to identify the c-Myc inhibitor compound 7594-0037 characteristically targeting c-Myc. However, Q-PCR results showed that compound 7594-0037 could not reduce $c-M y c$ mRNA 


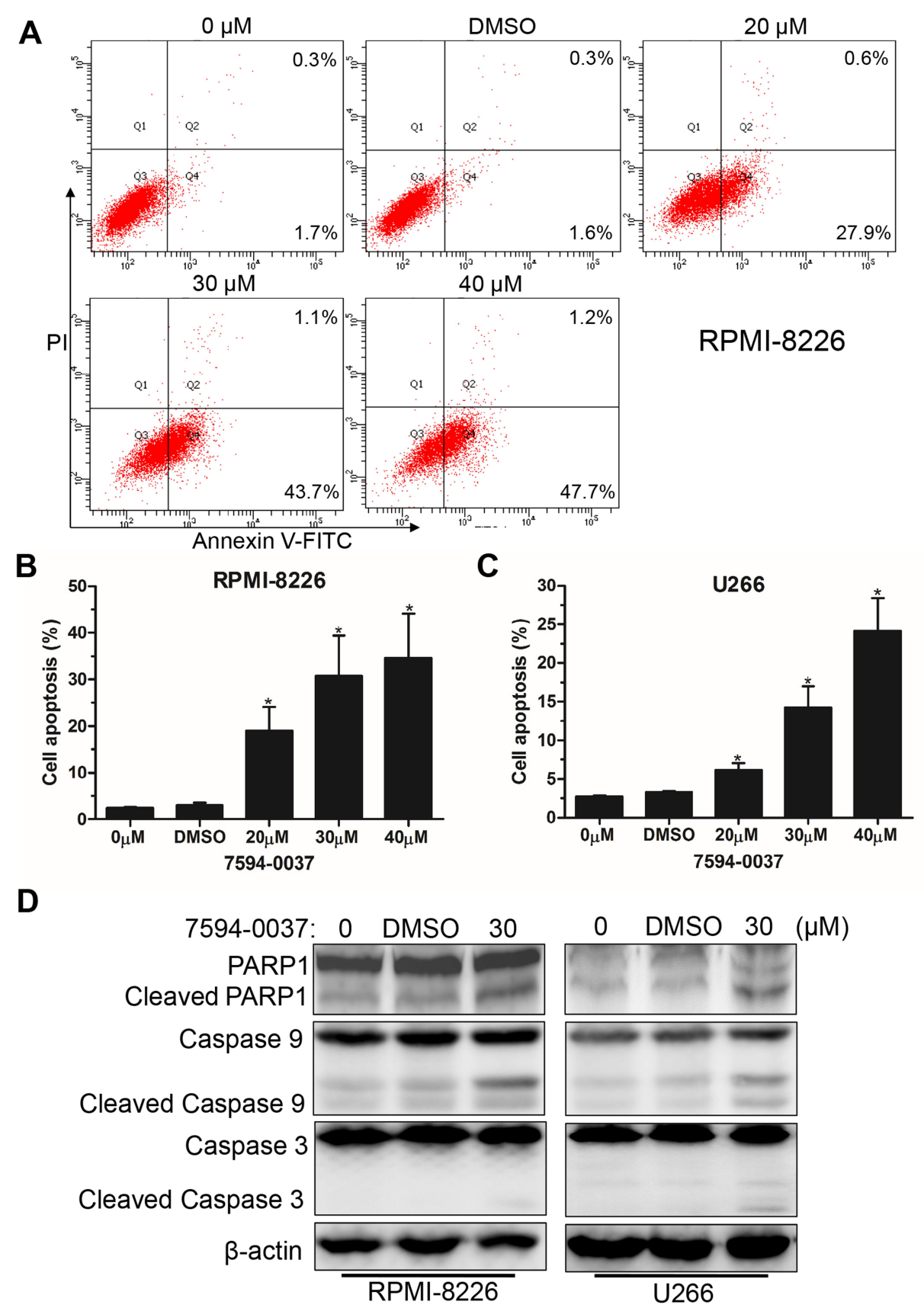

Figure 3 The effect of compound 7594-0037 on MM cell apoptosis. (A-B) RPMI-8226 cells treated with different doses of compound 7594-0037 were stained with Annexin V-FITC/PI, and flow cytometry analysis showed the ratio of apoptotic cells. (C) Percentages of U266 cell apoptosis based on three independent experiments of flow cytometry assay. (D) Western blot detection of apoptosis-associated PARPI, caspase-9, and caspase-3 proteins in RPMI-8226 and U266 cells treated with compound 7594-0037. Error bars: mean $\pm \mathrm{SD} ; * \mathrm{P}<0.05$.

levels in multiple MM cell lines (Figure 5A). To assess how compound 7594-0037 participates in c-Myc protein degradation, RPMI-8226 and U266 cells were treated using compound 7594-0037 at different times, and Western blot assay indicated that c-Myc protein expression decreased in a time-dependent manner following treatment with compound 7594-0037 (Figure 5B and $\mathrm{C}$ ). Due to the inherent high instability of the
c-Myc protein, ${ }^{36}$ we speculated that compound 7594-0037 may directly destroy c-Myc protein stability. Subsequently, we conducted protein stability assays using protein synthesis inhibitor cycloheximide (CHX) and found that compound 7594-0037 could rapidly facilitate c-Myc protein degradation after inhibition of protein synthesis (Figure 5D). Likewise, proteasome inhibitor MG132 reversed the c-Myc protein expression 

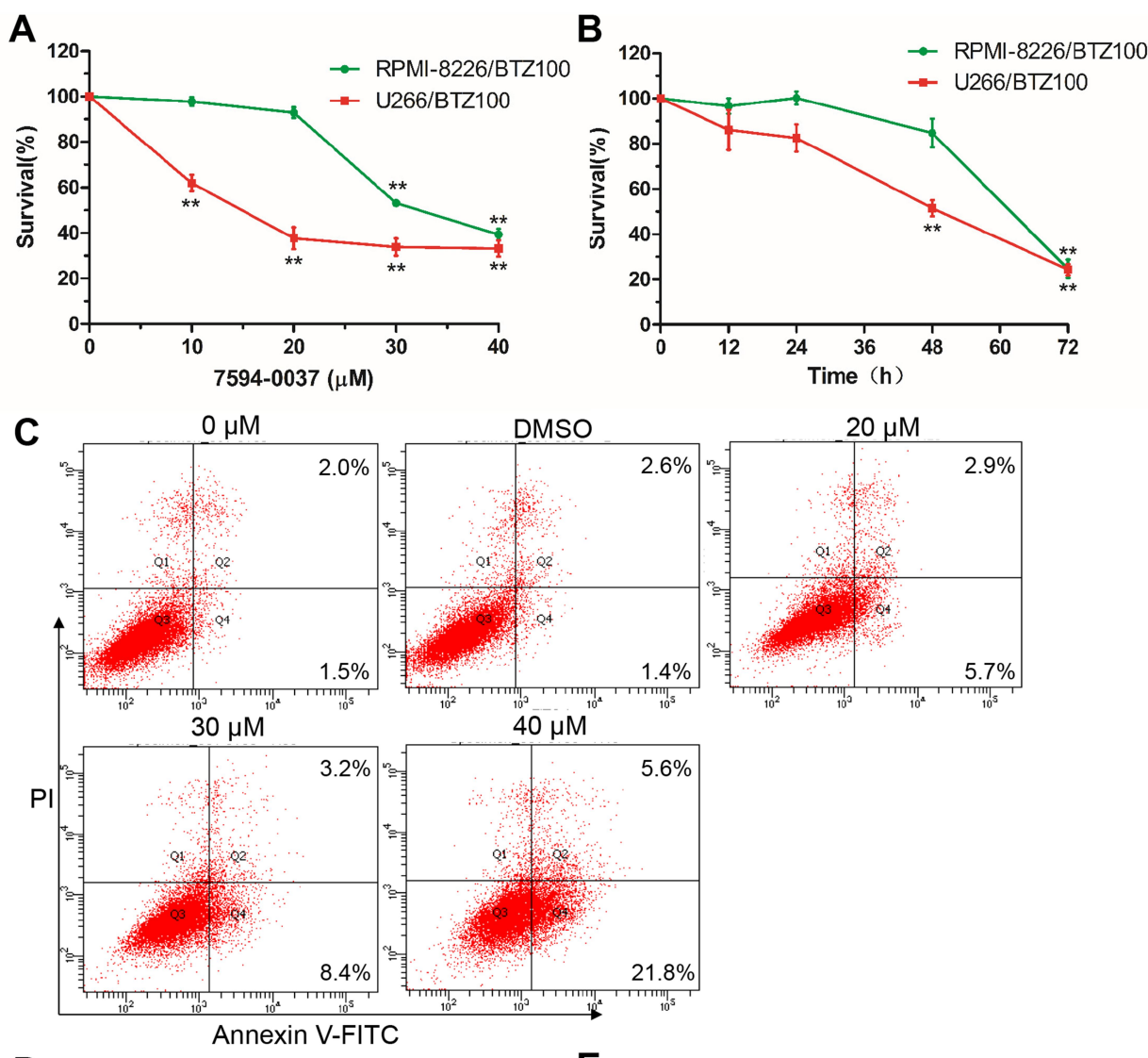

D

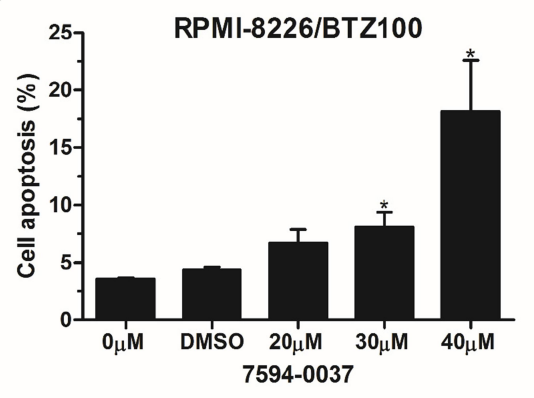

DMSO
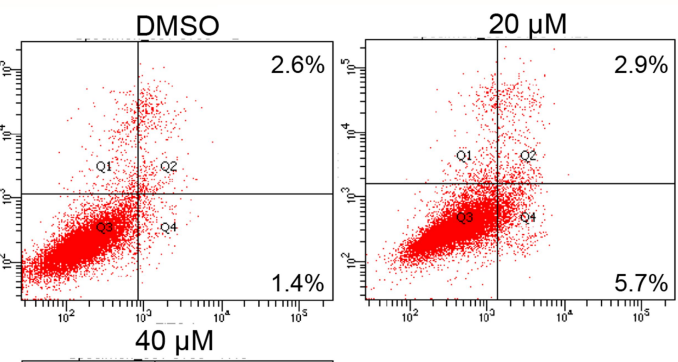
A

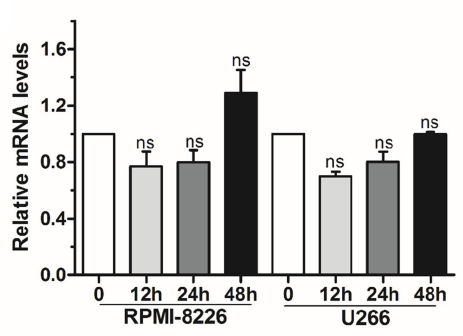

B

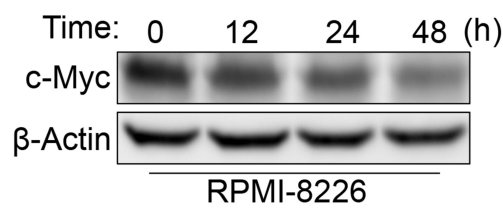

C

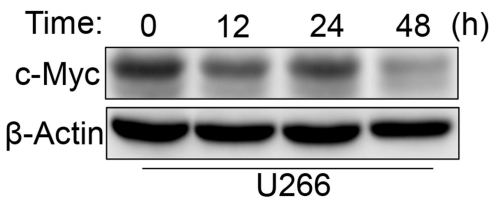

D

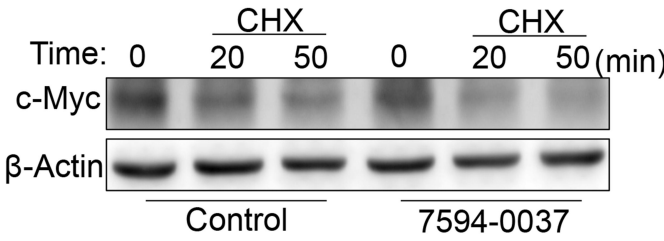

E

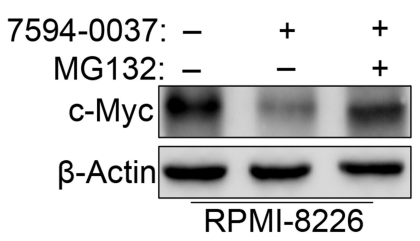

F
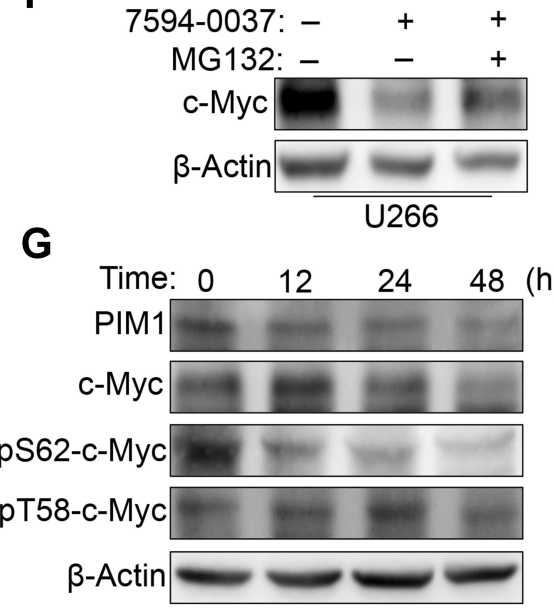

H

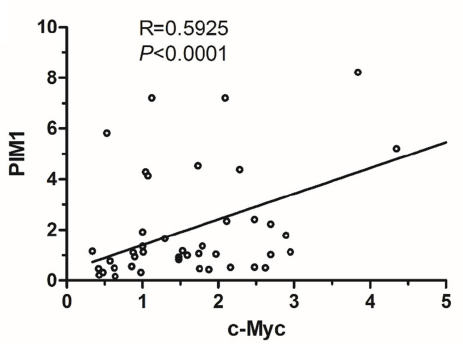

Figure 5 The effect of compound 7594-0037 on c-Myc protein stability. (A) The expression of c-Myc mRNA in RPMI-8226 and U266 cells was assessed by real-time PCR. ns, not significant. (B-C) Western blot analysis of c-Myc protein expression in RPMI-8226 and U266 cells treated with compound 7594-0037 at different time intervals. (D) U266 cells were treated with compound 7594-0037, following incubation with $\mathrm{CHX}$ for different amounts of time. Western blot detection of the change in c-Myc protein levels. (E-F) Western blot analyses of c-Myc expression in RPMI-8226 and U266 cells treated with compound 7594-0037 along with MGI32 treatment. (G) Western blot analysis of the expression levels of PIMI, c-Myc, pS62-c-Myc, and pT58-c-Myc in U266 cells via treatment with compound 7594-0037 for different amounts of time. (H) Pearson correlation analysis of PIMI and c-Myc mRNA levels in 44 newly diagnosed MM patients.

\section{Molecular Modeling of the Effect of} Compound 7594-0037 on c-Myc Protein

\section{Stability}

To further evaluate the effect of compound 7594-0037 on c-Myc protein stability, two c-Myc protein systems, ie, c-Myc/7594-0037 and c-Myc, were constructed and subjected to $20 \mathrm{~ns}$ molecular dynamics simulation. To explore the conformational stability of each system, the root mean square deviation (RMSD) of the protein backbone atoms was calculated with respect to the starting structure (Figure 6A). Overall, ligand-bound c-Myc protein seemed to be more deviated from the reference structure than ligand-free c-Myc (Figure 6B). Moreover, compound 7594-0037 decreased the stability of the C-terminus of
c-Myc rather than the N-terminus (Figure $6 \mathrm{C}$ and $\mathrm{D}$ ). This may be attributed to the fact that compound 7594-0037 bound to the N-terminus (Figure 6C) and could enhance the conformational stability of the N-terminus. As may be expected, the ligand-bound $\mathrm{N}$-terminus of c-Myc blocked the binding interactions between the $\mathrm{N}$ - and $\mathrm{C}$-termini, which resulted in the unconstrained state of the C-terminus of c-Myc.

To better characterize the effect of the binding of compound 7594-0037 on the interaction between the Nand $\mathrm{C}$-termini of c-Myc, the final configurations of the c-Myc protein in two systems after a 20-ns MD simulation are shown in Figure 7. In the ligand-free c-Myc system (Figure 7A), the residue Lys939 in the C-terminus formed one hydrogen bond with residue Leu917 in the 

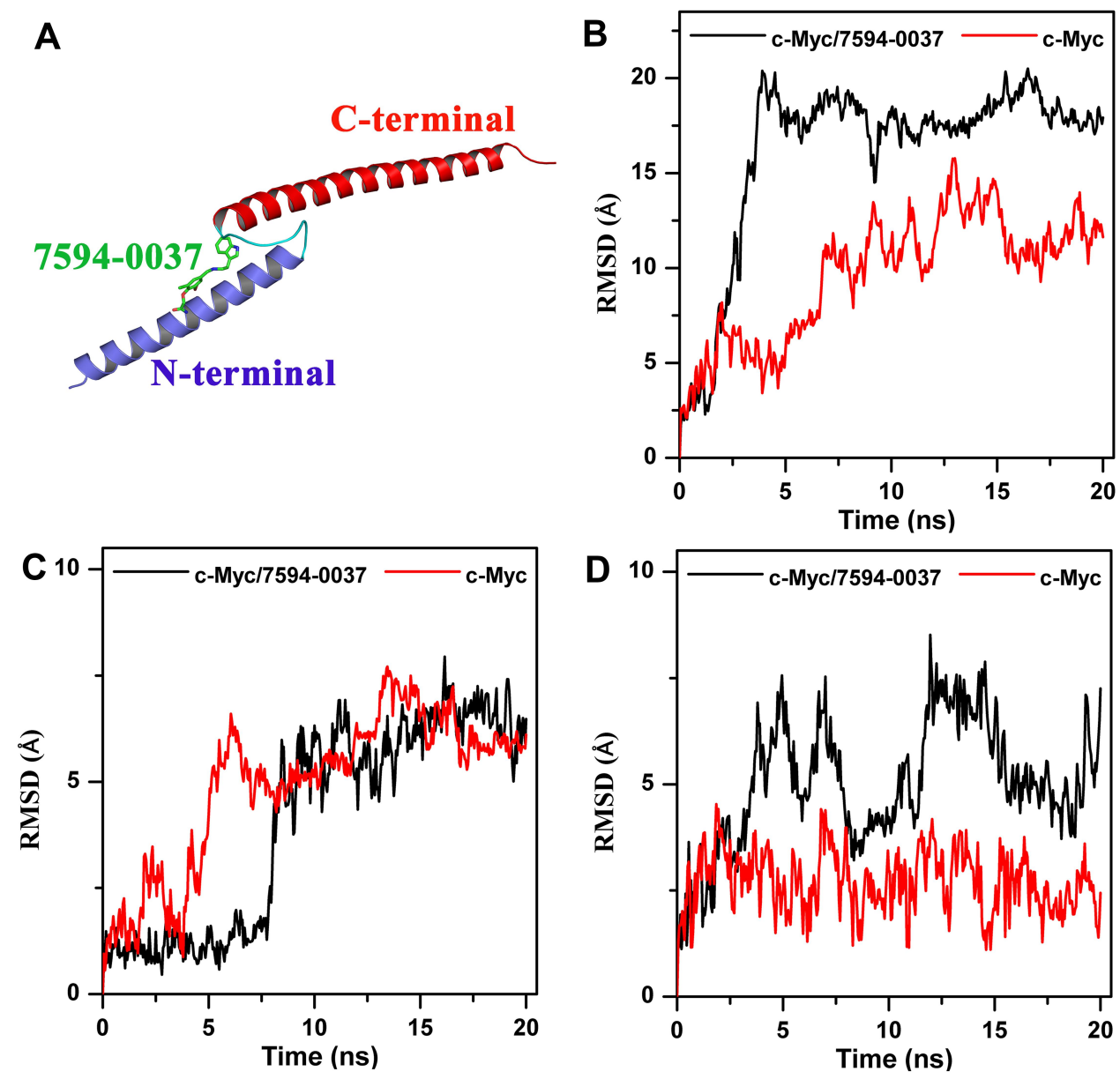

Figure 6 Molecular dynamics simulation to determine the effect of compound 7594-0037 on c-Myc protein stability. (A) Compound 7594-0037 interacted with the $\mathrm{N}$-terminus rather than the C-terminus of c-Myc protein. (B-D) RMSD of the backbone atoms of (B) c-Myc whole protein, (C) the N-terminus, and (D) the C-terminus with the reference structure at $\mathrm{t}=0 \mathrm{~ns}$ in two systems: c-Myc/7594-0037 (black) and single c-Myc protein (red).

N-terminus. Moreover, Lys939 exhibited strong hydrophobic interaction with Phe921. These interactions maintained the binding of the two termini of c-Myc. In the ligandbound c-Myc system (Figure 7B), the hydrogen bond that formed in two termini was missing, which may have been caused by the insertion of compound 7594-0037. At the same time, residue Phe921 interacted with the indole moiety of compound 7594-0037 rather than residue Lys939 in the C-terminus of c-Myc. Without any structural constraints, the C-terminus became more flexible. In total, compound 7594-0037 may affect c-Myc protein stability by interrupting the binding interactions of the two termini.

\section{Conclusion}

Multiple myeloma is one of the most frequent hematological cancers in the world. Currently, novel proteasome inhibitors (bortezomib) and immunomodulatory drugs (lenalidomide) have greatly improved MM patient survival, but $\mathrm{MM}$ remains incurable. The main reason for refractory $\mathrm{MM}$ is drug resistance, and therefore, it is urgent to explore novel molecular-targeted therapeutic agents. In this study, we identified a novel c-Myc inhibitor, compound 7594-0037, by structure based virtual screening, and it exhibited a stronger cytotoxic effect. Importantly, compound 7594-0037 could act to overcome myeloma bortezomib resistance and displayed a synergistic effect in combination with bortezomib.

As for the mechanism, in contrast to c-Myc inhibitor 10058-F4, compound 7594-0037 directly interfered with c-Myc protein stability and promoted c-Myc protein degradation via downregulated c-Myc S62 phosphorylation levels. The effect of compound 7594-0037 on c-Myc protein stability was also studied by molecular dynamics simulation. Once compound 7594-0037 was plugged into the $\mathrm{N}$-terminus of the c-Myc protein, the binding interactions (hydrogen bonds and hydrophobic interactions) between the 

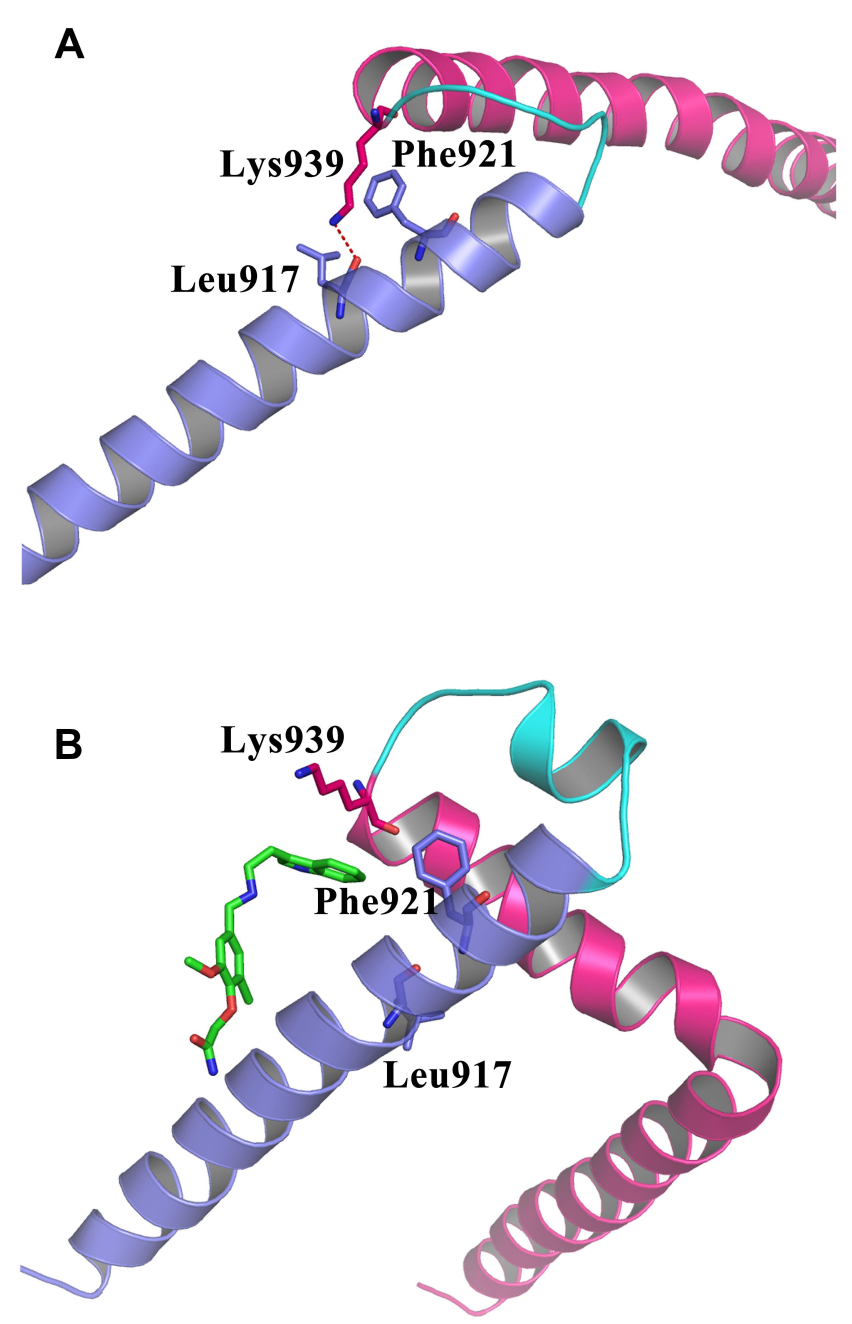

Figure 7 Compound 7594-0037 interrupted the binding interactions between the $\mathrm{N}$ - and $\mathrm{C}$-termini of c-Myc protein. (A) The detailed binding interactions at the two termini of c-Myc. (B) Compound 7594-0037 inserted into the interface of the two termini, and cleaved the hydrogen bond formed by residues Leu917 and Lys 939 . The $\mathrm{N}$ - and $\mathrm{C}$-termini of c-Myc are shown in cartoon models, and colored in blue and magenta, respectively.

$\mathrm{N}$ - and C-termini of c-Myc detached and the C-terminus would be more flexible. Taken together, our data demonstrate that novel c-Myc inhibitor compound 7594-0037 decreased MM cell survival and induced MM cell cycle G2 phase arrest. Additionally, compound 7594-0037 also visibly facilitated MM cell apoptosis. Therefore, our study highlights the clinical application potential of this novel c-Myc inhibitor.

\section{Abbreviations}

MM, multiple myeloma; PIM1, Proviral Insertion site in Moloney Murine Leukemia Virus (MMLV))1; bHLH-LZ, basic-helix-loop-helix leucine zipper; BRD4, bromodomain and extraterminal domain 4; RPMI, Roswell Park Memorial Institute; FBS, fetal bovine serum; PBS, phosphate-buffered saline; MD, molecular dynamics; PI, propidium iodide; BTZ, bortezomib; CHX, cycloheximide; RMSD, root mean square deviation.

\section{Acknowledgments}

This research was funded by he National Natural Science Foundation of China [grant number 81600173 and 21708033]; the Postdoctoral Science Foundation of China [grant numbers 2018T110554]; the Program for Distinguished Talents of Six Domains in Jiangsu Province (grant number YY-046) and the Qing Lan Project of Jiangsu Province; and Natural Science Foundation of the Jiangsu Higher Education Institutions of China (Grant No. 18KJB416008).

\section{Disclosure}

The authors report no conflicts of interest in this work.

\section{References}

1. Guo J, Hao J, Jiang H, et al. Proteasome activator subunit 3 promotes pancreatic cancer growth via c-Myc-glycolysis signaling axis. Cancer Lett. 2017;386:161-167. doi:10.1016/j.canlet.2016.08.018

2. Gomez-Curet I, Perkins RS, Bennett R, Feidler KL, Dunn SP, Krueger LJ. c-Myc inhibition negatively impacts lymphoma growth. J Pediatr Surg. 2006;41:207-211; discussion 207-211. doi:10.1016/j. jpedsurg.2005.10.025

3. Subramaniam KS, Omar IS, Kwong SC, et al. Cancer-associated fibroblasts promote endometrial cancer growth via activation of interleukin-6/STAT-3/c-Myc pathway. Am $J$ Cancer Res. 2016;6:200-213.

4. Pagnano KB, Vassallo J, Lorand-Metze I, Costa FF, Saad ST. p53, Mdm2, and c-Myc overexpression is associated with a poor prognosis in aggressive non-Hodgkin's lymphomas. Am $J$ Hematol. 2001;67:84-92. doi:10.1002/ajh.1084

5. McNeil CM, Sergio CM, Anderson LR, et al. c-Myc overexpression and endocrine resistance in breast cancer. J Steroid Biochem Mol Biol. 2006;102:147-155. doi:10.1016/j.jsbmb.2006.09.028

6. Prochownik EV, Vogt PK. Therapeutic Targeting of Myc. Genes Cancer. 2010;1:650-659. doi:10.1177/1947601910377494

7. Hu Y, Yu K, Wang G, et al. Lanatoside C inhibits cell proliferation and induces apoptosis through attenuating Wnt/ $\beta$-catenin/c-Myc signaling pathway in human gastric cancer cell. Biochem Pharmacol. 2018;150:280-292. doi:10.1016/j.bcp.2018.02.023

8. Chng WJ, Huang GF, Chung TH, et al. Clinical and biological implications of MYC activation: a common difference between MGUS and newly diagnosed multiple myeloma. Leukemia. 2011;25:1026-1035. doi:10.1038/leu.2011.53

9. Szabo AG, Gang AO, Pedersen MO, Poulsen TS, Klausen TW, Norgaard P. Overexpression of c-myc is associated with adverse clinical features and worse overall survival in multiple myeloma. Leuk Lymphoma. 2016;57:2526-2534. doi:10.1080/10428194.2016. 1187275

10. Fletcher S, Prochownik EV. Small-molecule inhibitors of the Myc oncoprotein. Biochim Biophys Acta. 2015;1849:525-543. doi:10.1016/j.bbagrm.2014.03.005

11. Delmore JE, Issa GC, Lemieux ME, et al. BET bromodomain inhibition as a therapeutic strategy to target c-Myc. Cell. 2011;146:904-917. doi:10.1016/j.cell.2011.08.017 
12. Morello D, Asselin C, Lavenu A, Marcu KB, Babinet C. Tissue-specific post-transcriptional regulation of c-myc expression in normal and $\mathrm{H}-2 \mathrm{~K}$ / human c-myc transgenic mice. Oncogene. 1989;4:955-961.

13. Gregory MA, Qi Y, Hann SR. Phosphorylation by glycogen synthase kinase-3 controls c-myc proteolysis and subnuclear localization. J Biol Chem. 2003;278:51606-51612. doi:10.1074/jbc.M310722200

14. Zhang Y, Wang Z, Li X, Magnuson NS. Pim kinase-dependent inhibition of c-Myc degradation. Oncogene. 2008;27:4809-4819. doi:10.1038/onc.2008.123

15. Welcker M, Orian A, Jin J, et al. The Fbw7 tumor suppressor regulates glycogen synthase kinase 3 phosphorylation-dependent c-Myc protein degradation. Proc Natl Acad Sci $U S A$. 2004;101:9085-9090. doi:10.1073/pnas.0402770101

16. Farrell AS, Sears RC. MYC degradation. Cold Spring Harb Perspect Med. 2014;4.

17. Nair SK, Burley SK. X-ray structures of myc-max and mad-max recognizing DNA: molecular bases of regulation by proto-oncogenic transcription factors. Cell. 2003;112:193-205. doi:10.1016/S0092-8674(02)01284-9

18. Franke NE, Niewerth D, Assaraf YG, et al. Impaired bortezomib binding to mutant beta5 subunit of the proteasome is the underlying basis for bortezomib resistance in leukemia cells. Leukemia. 2012;26:757-768. doi:10.1038/leu.2011.256

19. Mei PJ, Bai J, Miao FA, et al. Relationship between expression of $\mathrm{XRCC} 1$ and tumor proliferation, migration, invasion, and angiogenesis in glioma. Invest New Drugs. 2018.

20. Wang T, Pan D, Zhang Y, et al. Luteolin antagonizes angiotensin II-dependent proliferation and collagen synthesis of cultured rat cardiac fibroblasts. Curr Pharm Biotechnol. 2015;16:430-439. doi:10.2174/1389201015666141110142402

21. Cao WJ, Mao LL, Zheng JN, Pei DS. p42.3: an abductor of cell cycle. Anticancer Agents Med Chem. 2015;15:157-162. doi:10.2174/ 1871520614666140818200202

22. Yao R, Han D, Sun X, et al. Scriptaid inhibits cell survival, cell cycle, and promotes apoptosis in multiple myeloma via epigenetic regulation of p21. Exp Hematol. 2018;60:63-72. doi:10.1016/j. exphem.2017.12.012

23. Ji S, Tang S, Li K, et al. Licoricidin inhibits the growth of SW480 human colorectal adenocarcinoma cells in vitro and in vivo by inducing cycle arrest, apoptosis and autophagy. Toxicol Appl Pharmacol. 2017;326:25-33. doi:10.1016/j.taap.2017.04.015

24. Li L, Xu T, Du Y, et al. Salvianolic Acid A attenuates cell apoptosis, oxidative stress, Akt and NF-kappaB activation in Angiotensin-II induced murine peritoneal macrophages. Curr Pharm Biotechnol. 2016;17:283-290. doi:10.2174/138920101703160206150535
25. Liu L, Sun X, Xie Y, Zhuang Y, Yao R, Xu K. Anti-proliferative activity of HPOB against multiple myeloma cells via p21 transcriptional activation. Molecules. 2018;23.

26. Yao R, Sun X, Xie Y, et al. Identification of a novel c-Myc inhibitor with antitumor effects on multiple myeloma cells. Biosci Rep. 2018;38.

27. Case DA, Cheatham TE 3rd, Darden T, et al. The Amber biomolecular simulation programs. J Comput Chem. 2005;26:1668-1688. doi: $10.1002 /$ jcc. 20290

28. Frisch MJ, Trucks GW, Schlegel HB, et al. Gaussian 09 Rev. A.02. Wallingford, CT; 2016.

29. Gao J, Zhang Y, Chen $\mathrm{H}$, et al. Computational insights into the interaction mechanism of transcription cofactor vestigial-like protein 4 binding to TEA domain transcription factor 4 by molecular dynamics simulation and molecular mechanics generalized Born/surface area) calculation. J Biomol Struct Dyn. 2019;37(10):2538-2545. doi:10.1080/07391102.2018.1491889

30. Chen H, Wang Y, Gao Z, Yang W, Gao J. Assessing the performance of three resveratrol in binding with SIRT1 by molecular dynamics simulation and MM/GBSA methods: the weakest binding of resveratrol 3 to SIRT1 triggers a possibility of dissociation from its binding site. J Comput-Aided Mol Des. 2019;33:437-446. doi:10.1007/ s10822-019-00193-0

31. Gao J, Liang L, Chen Q, Zhang L, Huang T. Insight into the molecular mechanism of yeast acetyl-coenzyme A carboxylase mutants F510I, N485G, I69E, E477R, and K73R resistant to soraphen A. J Comput-Aided Mol Des. 2018;32:547-557. doi:10.1007/s10822018-0108-z

32. Yin X, Giap C, Lazo JS, Prochownik EV. Low molecular weight inhibitors of Myc-Max interaction and function. Oncogene. 2003;22:6151-6159. doi:10.1038/sj.onc.1206641

33. Wang H, Hammoudeh DI, Follis AV, et al. Improved low molecular weight Myc-Max inhibitors. Mol Cancer Ther. 2007;6:2399-2408. doi:10.1158/1535-7163.MCT-07-0005

34. Holien T, Vatsveen TK, Hella H, Waage A, Sundan A. Addiction to c-MYC in multiple myeloma. Blood. 2012;120:2450-2453. doi:10.1182/blood-2011-08-371567

35. Adachi Y, Yoshio-Hoshino N, Nishimoto N. Gene therapy for multiple myeloma. Curr Gene Ther. 2008;8:247-255. doi:10.2174/ 156652308785160683

36. Thomas LR, Tansey WP. Proteolytic control of the oncoprotein transcription factor Myc. Adv Cancer Res. 2011;110:77-106.

\section{Publish your work in this journal}

Drug Design, Development and Therapy is an international, peerreviewed open-access journal that spans the spectrum of drug design and development through to clinical applications. Clinical outcomes, patient safety, and programs for the development and effective, safe, and sustained use of medicines are a feature of the journal, which has also been accepted for indexing on PubMed Central. The manuscript management system is completely online and includes a very quick and fair peer-review system, which is all easy to use. Visit http://www. dovepress.com/testimonials.php to read real quotes from published authors. 